\title{
Sequential Dramatic Regrowth of a Lumbar Pedicle Post-Antitubercular Therapy
}

\author{
Saurabh Kumar Verma ${ }^{1}$ Giriraj Singh ${ }^{2}$ Neeraj Basantani ${ }^{3} \quad$ Amit Narang $^{3}$ \\ ${ }^{1}$ Department of Neurosurgery, Army Hospital (R\&R), Delhi Cantt, \\ New Delhi, Delhi, India \\ ${ }^{2}$ Command Hospital (SC), Pune, India \\ ${ }^{3}$ Department of Neurosurgery, Armed Forces Medical College, Pune, India \\ Address for correspondence Saurabh Kumar Verma, MCh, \\ Department of Neurosurgery, Army Hospital (R\&R), New Delhi, \\ Delhi 110010, India (e-mail: eskay6362@yahoo.co.in). \\ Indian J Neurosurg 2016;5:213-215.
}

\begin{abstract}
Keywords

- lumbar pedicle

- tuberculosis

- antitubercular therapy

Spinal tuberculosis is a frequently encountered extrapulmonary form of the disease. Despite this disease being rampant in developing countries, there are no straightforward guidelines for the diagnosis and treatment of spinal tuberculosis. Surgical intervention is deemed necessary in advanced cases with marked bony involvement or when the spine is considered potentially unstable. However, with the modernization of spinal instrumentation, the general trend among spine surgeons is to fix even a potentially unstable spine. The dilemma still remains whether such cases should be managed surgically or they can be successfully treated using more accurate diagnostic methods and antitubercular therapy (ATT). Here we report the case of a young man with lumbar pedicle tuberculosis who was successfully diagnosed and managed with percutaneous biopsy and ATT vis-à-vis open surgery and fixation. Dramatic resolution of the disease process over a period of 12 months on ATT in this case has been highlighted, thus avoiding fixation in a potentially unstable spine.
\end{abstract}

\section{Introduction}

Spinal tuberculosis is a frequently encountered extrapulmonary form of the disease. Despite this disease being rampant in developing countries, there are no straightforward guidelines for the diagnosis and treatment of spinal tuberculosis. ${ }^{1}$ Indications for surgery as suggested by an expert were panvertebral lesions, refractory disease, that is, failure of chemotherapy after 3 to 4 weeks, severe kyphosis, an evolving neurological deficit, and clinical deterioration or lack of clinical improvement. ${ }^{2}$ Also, pedicle involvement in tuberculosis has been considered by many surgeons to be potentially unstable and hence is managed operatively. However, there is no clear-cut definition of a potentially unstable lumbar spine. Campbell et al highlighted the role of computed tomography (CT) scan in identifying potentially unstable lumbar fractures and included any middle column or posterior column injuries (except transverse process and

received

December 22, 2015 accepted after revision January 14, 2016 published online June 8, 2016
DOI http://dx.doi.org/

10.1055/s-0036-1581975. ISSN 2277-954X. spinous process) as potentially unstable. ${ }^{3}$ Here, we report the case of a 22-year-old man who presented with worsening low back pain of 1-year duration and a diagnostic and therapeutic dilemma on imaging which was managed successfully with CT-guided biopsy and antitubercular therapy (ATT), highlighting the importance of CT-guided biopsy in the diagnosis of Pott spine and timely ATT. Moreover, a complete regrowth of an eaten-up pedicle with ATT alone has not been demonstrated, thus precluding the need for surgery in such cases.

\section{Case Summary}

An immunocompetent 22-year-old man presented with worsening low back pain of 1-year duration without any history of radicular pain, neurogenic claudication, weakness of lower limbs, sphincter disturbances, cough, fever, night sweats, or trauma. He had associated unquantified weight (c) 2016 Neurological Surgeons' Society of India
License terms

(요 (1) $\Theta \circledast$ 


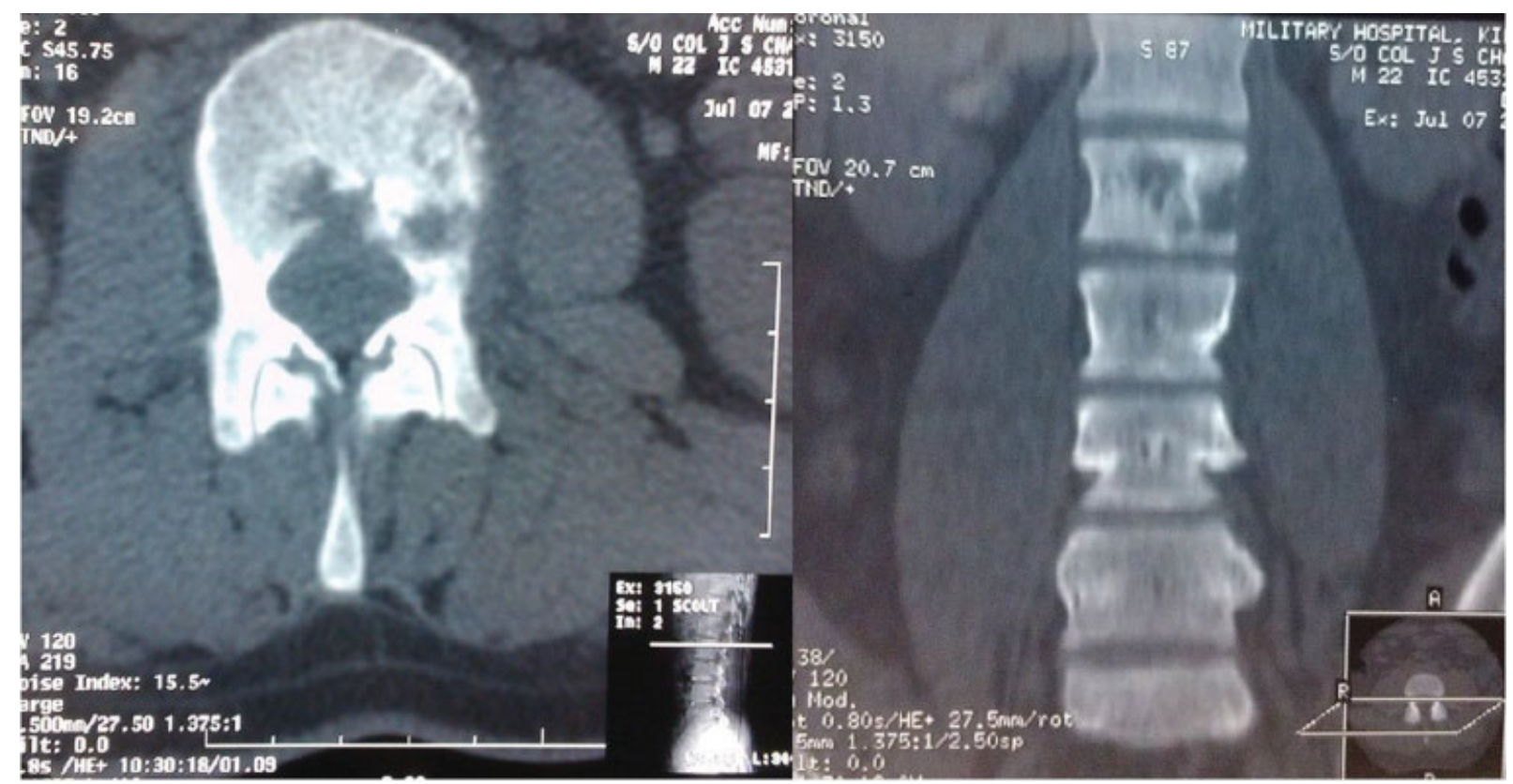

Fig. 1 Left-hand panel: NCCT lumbar spine axial view at LV2 level showing lytic lesions in the vertebral body and left pedicle. Right-hand panel: coronal view showing lytic lesions in the vertebral body.

loss and decreased appetite. Neurological examination revealed SLR restricted to 60 degrees bilaterally. However, there was no spinal deformity or tenderness, no weakness of lower limbs, and no sensory deficits, and plantars were flexor bilaterally and gait was normal.

Plain radiograph of the lumbosacral spine showed lytic lesion in the left LV2 pedicle and body. NCCT of the lumbar spine showed lytic lesions in the LV2 vertebral body and almost complete destruction of the left LV2 pedicle ( - Fig. 1). Contrastenhanced magnetic resonance imaging (CEMRI) of the lumbar spine revealed postcontrast enhancement of LV2 vertebral body along with the left LV2 pedicle with minimal left paravertebral soft tissue enhancement. CT-guided trucut biopsy of the L2 lytic lesion was done, which was negative in the first instance. In view of the clinicoradiological picture strongly suggestive of an infective process, decision was taken to repeat the CT-guided biopsy. The second biopsy pathognomically demonstrated necrotizing tubercular granulomas.

Once the diagnosis was confirmed to be of tubercular etiology, the patient was started on four-drug regimen of ATT with bed rest and followed-up, with LFT once a month and NCCT of the lumbar spine thrice a month. The patient improved symptomatically with decreased low back pain and weight gain gradually. Follow-up imaging was suggestive of resolution of the lesion. Presently, the patient has completed 15 months of ATT, is ambulant without any neurological deficits, and is on regular follow-up. Sequential NCCT images, over the course of ATT, have shown dramatic regrowth of the "eaten-up" left LV2 pedicle (-Fig. 2).

\section{Discussion}

Chronic back pain as the only symptom was observed in $61 \%$ of cases of spinal tuberculosis, as in our case. ${ }^{4}$ In view of his uncommon radiological findings involving the pedicle, the authors were prompted to confirm the diagnosis with a CT-guided biopsy. Paradiscal, anterior, and central lesions are the common types of vertebral

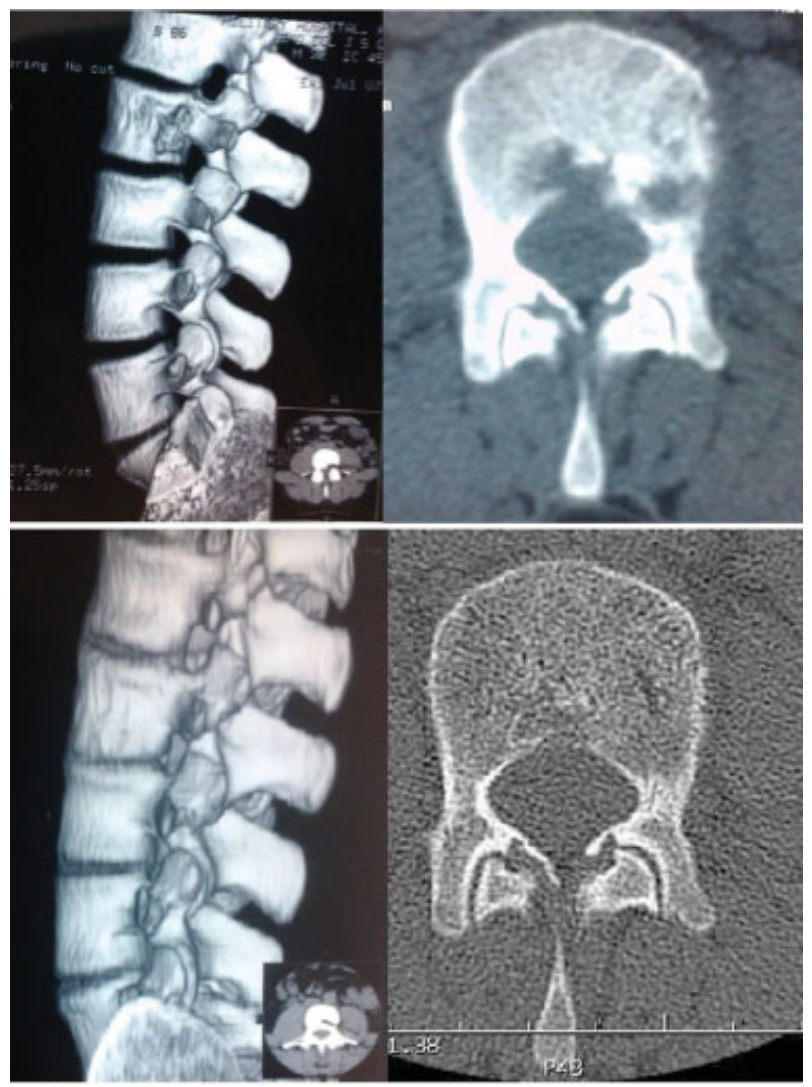

Fig. 2 Upper panel: lytic lesions of body and left pedicle in 3D sagittal and axial views. Lower panel: comparative images showing significant resolution of lesions after 6 months of treatment. 
involvement, pedicular involvement alone being rare in spinal tuberculosis. ${ }^{5}$ As the first CT-guided biopsy was inconclusive, a repeat biopsy was done considering the patients clinical profile. Neuroimaging-guided needle biopsy from the affected site is the gold standard technique for the early histopathological diagnosis of spinal tuberculosis. ${ }^{6}$ Surgery may be required in up to $10 \%$ of cases to establish the etiological diagnosis, even when achieving stability is not the goal of the surgeon. ${ }^{4}$ Once the diagnosis of spinal tuberculosis was confirmed on biopsy, the patient was started on ATT for a period of 18 months as suggested by Jain et al. ${ }^{1}$ Follow-up of the patient with three CT scans per month showed dramatic regrowth of the pedicle, as depicted earlier. Presently, the patient is in his 15th month of ATT, is essentially asymptomatic, has gained weight, and has no neurological deficits.

Tuli, in 1975, proposed a "middle-path regimen" for treatment of spinal tuberculosis. It advocates conservative treatment with multidrug chemotherapy, with surgery reserved for specific indications. ${ }^{7}$ Indications for surgery were panvertebral lesions, refractory disease, severe kyphosis, an evolving neurological deficit, and clinical deterioration or lack of clinical improvement. ${ }^{8}$ These indications still hold true, but with more and more patients approaching the spine surgeon with CT and MRI films, the authors feel that indications need to be reconsidered. Further studies to classify what is potentially unstable spine are required, to relieve the neurosurgeons from the dilemma of whether to operate upon potentially unstable spine in extrapulmonary tuberculosis patients.

\section{Conclusion}

A high degree of clinical suspicion is required if patients present with chronic back pain, even in the absence of neurological symptoms and signs. A meticulous attempt must be made to diagnose spinal tuberculosis histopathologically, in cases with highly suspicious radiology findings. Morbidity and risks of spinal surgery can be avoided even in potentially unstable spines with prolonged ATT, as illustrated by our case. Future randomized controlled trials can be directed toward comparison of medical versus surgical treatment in such cases.

\section{Acknowledgment}

The authors thank the Department of Pathology, Command Hospital (Southern Command), Pune.

\section{References}

1 Jain AK. Tuberculosis of the spine: a fresh look at an old disease. J Bone Joint Surg Br 2010;92(7):905-913

2 Sell P. Expert's comment concerning Grand Rounds case entitled "Posterior listhesis of a lumbar vertebra in spinal tuberculosis" (by Matthew A. Kirkman and Krishnamurthy Sridhar). Eur Spine J 2011;20(1):6-8

3 Campbell SE, Phillips CD, Dubovsky E, Cail WS, Omary RA. The value of $\mathrm{CT}$ in determining potential instability of simple wedgecompression fractures of the lumbar spine. AJNR Am J Neuroradiol 1995;16(7):1385-1392

4 Cormican L, Hammal R, Messenger J, Milburn HJ. Current difficulties in the diagnosis and management of spinal tuberculosis. Postgrad Med J 2006;82(963):46-51

5 Shanley DJ. Tuberculosis of the spine: imaging features. AJR Am J Roentgenol 1995;164(3):659-664

6 Jain R, Sawhney S, Berry M. Computed tomography of vertebral tuberculosis: patterns of bone destruction. Clin Radiol 1993; 47(3):196-199

7 Tuli SM. Results of treatment of spinal tuberculosis by "middlepath" regime. J Bone Joint Surg Br 1975;57(1):13-23

8 Jutte PC, Castelein RM. Complications of pedicle screws in lumbar and lumbosacral fusions in 105 consecutive primary operations. Eur Spine J 2002;11(6):594-598 